
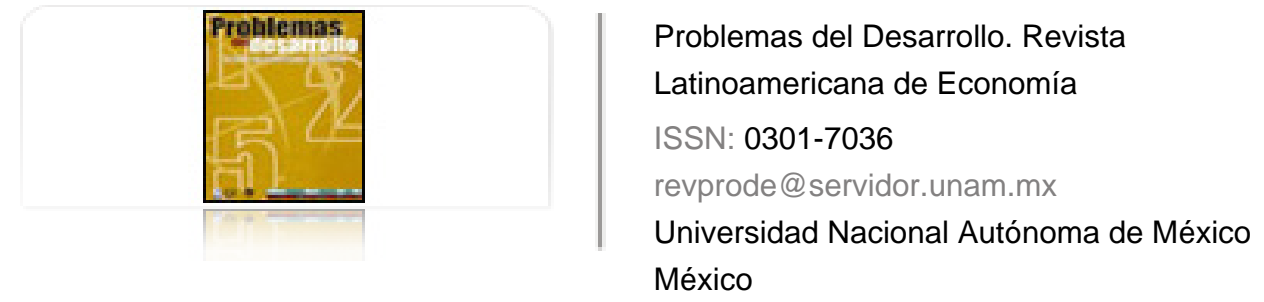

Allami, Cecilia; Cibils, Alan

El financiamiento bancario de las PYMES en Argentina (2002 - 2009)

Problemas del Desarrollo. Revista Latinoamericana de Economía, vol. 42, núm. 165, abril-junio, 2011, pp. 61-86

Universidad Nacional Autónoma de México

Distrito Federal, México

Disponible en: http://www.redalyc.org/articulo.oa?id=11819780004

Cómo citar el artículo

Número completo

- Más información del artículo

- Página de la revista en redalyc.org

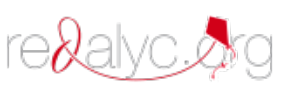

Sistema de Información Científica

Red de Revistas Científicas de América Latina, el Caribe, España y Portugal Proyecto académico sin fines de lucro, desarrollado bajo la iniciativa de acceso abierto 


\title{
EL FINANCIAMIENTO BANCARIO DE LAS PYMES en Argentina (2002-2009)
}

\author{
Cecilia Allami y Alan Cibils*
}

Fecha de recepción: 24 de noviembre de 2010. Fecha de aceptación: 8 de marzo de 2011 .

\section{RESUMEN}

Entre 2003 y 2007, la economía argentina experimentó una de las fases de crecimiento más intensas y extensas de las últimas décadas. En este nuevo escenario, las pequeñas y medianas empresas (PYMEs) tuvieron un notable desempeño económico y recuperaron el dinamismo que habían perdido en la década anterior. No obstante, la limitación de acceso a los mercados de crédito por parte de las PYMEs en Argentina es un fenómeno ampliamente reconocido, que compromete las posibilidades de crecimiento y expansión de estas empresas. Este trabajo analiza el crédito bancario efectivamente otorgado a las PYMEs en el periodo 2002-2009 en Argentina. Se concluye la necesidad de intervención y regulación del Estado argentino en el mercado bancario, así como la redefinición de las políticas de crédito de entidades públicas y otras políticas focalizadas para mejorar el financiamiento de estas empresas. Palabras clave: crédito, estructuralismo, financiamiento del desarrollo, pequeñas y medianas empresas, sistema bancario.

\section{BANK FINANCING OF SMALL AND MEDIUM-SIZED COMPANIES IN Argentina (2002 - 2009)}

\section{Abstract}

From 2003 to 2007, the Argentine economy experienced one of its most intensive and extensive phases of growth in recent decades. In this new scenario, small and mediumsized companies (PYMEs) registered notable economic performance and recovered the dynamism they had lost in the previous decade. Nevertheless, the Argentine PYMEs' limited access to credit markets is a widely recognized phenomenon, which limits the possibilities for the growth and expansion of these companies. This paper analyzes the bank credit effectively extended to the PYMEs during the period 2002-2009 in Argentina. It concludes that there is a need for intervention and regulation by the Argentine State in the banking market, as well as a redefinition of credit policies for public entities and other policies focused on improving the financing of these companies.

Key words: credit, structuralism, development finance, small and medium-sized companies, banking system.

* Investigadores-docentes del Área de Economía Política del Instituto de Industria de la Universidad Nacional de General Sarmiento (UnGs), Argentina. Correo electrónico: callami@ ungs.edu.ary acibils@ungs.edu.ar 


\section{LE FINANCEMENT BANCAIRE DES PME EN ARGENTINE (2002-2009) Résumé}

Entre 2003 et 2007, l'économie argentine a connu une des phases de croissance les plus intenses et longues des dernières décennies. Dans ce nouveau panorama économique, les petites et moyennes entreprises (PME) ont été particulièrement actives et ont récupéré le dynamisme qu'elles avaient perdu au cours de la décennie précédente. Néanmoins, la limitation de l'accès aux marchés de crédit pour les PME en Argentine est un phénomène largement reconnu et qui compromet les possibilités de croissance et d'expansion de ces entreprises. Ce travail analyse le crédit bancaire effectivement octroyé aux PME entre 2002 et 2009 en Argentine. Il conclut à la nécessité que l'État argentin intervienne et régule le marché bancaire tout en redéfinissant les politiques de crédit des organismes publics et d'autres politiques appropriées pour améliorer le financement de ces entreprises. Mots clés : crédit, structuralisme, financement du développement, petites et moyennes entreprises, système bancaire.

\section{O FINANCIAMENTO BANCÁRIO DAS PMES NA ARGENTINA (2002-2009) Resumo}

Entre 2003 e 2007, a economia argentina experimentou uma das fases de crescimento mais intensas e extensas das últimas décadas. Neste novo cenário, as pequenas e médias empresas (PMEs) tiveram um notável desempenho econômico e recuperaram o dinamismo que tinham perdido na década anterior. Não obstante, a limitação de acesso aos mercados de créditos por parte das pMEs na Argentina é um fenômeno amplamente reconhecido, que compromete as possibilidades de crescimento e expansão destas empresas. Este trabalho analisa o crédito bancário efetivamente outorgado às PMEs no período 2002-2009 na Argentina. Conclui-se a necessidade de intervenção e regulação do Estado argentino no mercado bancário, assim como a redefinição das políticas de crédito de entidades públicas e outras políticas focalizadas para melhorar o financiamento destas empresas.

Palavras-chave: Crédito, Estruturalismo, Financiamento do desenvolvimento, Pequenas e médias empresas, Sistema bancário.

阿根廷中小企业银行融资(2002-2009)

塞西莉亚・阿亚米及阿兰・西比尔斯

概要

2003 至2007年间, 阿根廷经济经历了最近几十年来最强劲而广泛的增长 期。在这个全新的舞台上, 中小企业的经济表现引人瞩目, 找回了之前一 个十年所失去的活力。然而, 阿根廷中小企业进入信贷市场所受到的限制 广为人知, 这种限制影响了它们成长和扩张的可能。这份报告分析了 2002 至2009年间阿根廷真正下放给中小企业的银行信贷，总结出阿根廷政府介 入并规范其银行市场的必要性, 同时指出应当重新定义公共领域信贷政策 以及其他为改善这些企业融资而专门制定的政策。

关键词：信贷, 结构主义, 发展融资, 中小企业, 银行系统 


\section{INTRODUCCIÓN}

Como consecuencia de la aplicación de un drástico paquete de reformas promercado, durante la década del 90 se inició un proceso de profundización de las transformaciones estructurales en la economía argentina iniciadas por la dictadura en el año 1976. La fijación del tipo de cambio y la convertibilidad monetaria, la apertura comercial y financiera, la desregulación económica y la descentralización de diversas actividades estatales -entre otras medidas- determinaron un particular comportamiento de la economía argentina durante la década y generaron profundos cambios en la estructura económica. En este marco, la desarticulación del entramado industrial afectó particularmente a las pequeñas y medianas empresas (PYMEs). Este tipo de unidades productivas, por poseer -en líneas generales- una menor capacidad financiera, tecnológica y de presión política respecto a las grandes, tuvo mayores dificultades para adaptarse a las transformaciones en curso ${ }^{1}$ (Fernández Bugna y Porta, 2008). Por otra parte, el proceso de concentración ${ }^{2}$ y extranjerización del sistema bancario durante la década del $90^{3}$-junto con las restrictivas normas de previsión y de calificación de empresas ${ }^{4}$ generó fuertes cambios en el patrón de financiamiento de la producción, debido a la desaparición de bancos tradicionalmente ligados a financiar el segmento PYME (como los bancos cooperativos y los bancos públicos provinciales y municipales) y el impacto de los procesos de fusión en el mercado de préstamos, que castigaron aún más a los pequeños y medianos empresarios tanto en términos de cantidad de créditos otorgados como de las altas tasas de interés aplicadas (Bleger y Rozenwurcel, 2007; Damill et al, 2004).

Tras la larga recesión iniciada a mediados de 1998, el posterior colapso del régimen de convertibilidad a fines de 2001 y la crítica situación del año 2002, la

1 Durante esa década, las PYMEs retrocedieron tanto en términos de su aporte al PIB como al empleo. Además, fueron vulnerables a las fluctuaciones macroeconómicas y a la mayor exposición a la competencia internacional generada por las medidas de liberalización y de mayor apertura económica (BID, 2002).

2 Concentración tanto respecto al número de bancos como de depósitos y préstamos en pocas entidades.

3 Véase Cibils y Allami (2010).

4 La adopción de regulaciones prudenciales de acuerdo con las normas del Comité de Basilea que consistió principalmente en la imposición de requerimientos mínimos de capital vinculados al riesgo de los activos de los bancos- potenció las condiciones para el proceso de racionamiento del crédito destinado a los proyectos encarados por las pequeñas y medianas empresas (Wierzba y Golla, 2005). 
economía argentina comenzó a mostrar fuertes señales de recuperación. Entre 2003 y 2007, la economía argentina experimentó una de las fases de crecimiento más intensas y extensas de las últimas décadas, con un crecimiento promedio cercano al $9 \%$ anual. $\mathrm{Al}$ mismo tiempo, el desempeño económico estuvo caracterizado por una fuerte expansión del mercado interno, la sustitución de importaciones, la expansión de la inversión y las exportaciones y el incremento del empleo (Frenkel, 2005; Kulfas, 2008).

En este nuevo escenario, las PYMEs tuvieron un notable desempeño económico y recuperaron el dinamismo que habían perdido en la década anterior. Su aporte al crecimiento fue muy relevante en términos de empleo, inversión, nivel de actividad y, en menor medida, exportaciones (Fundación Observatorio PYME, 2003-2008). No obstante, persisten otros desafíos entre los que se destaca el financiamiento de la inversión. La limitación de acceso a los mercados de crédito por parte de las PYMEs en Argentina es un fenómeno ampliamente reconocido (Ascúa, 2010; Fundación Observatorio pyme, 2010). Se trata de un problema de gran relevancia, en la medida en que las restricciones al financiamiento podrían afectar a la inversión, comprometiendo las posibilidades de crecimiento y las ganancias de productividad necesarias para la consolidación y expansión de estas empresas. Adicionalmente, cabe destacar que en los países en vías de desarrollo, el crédito bancario constituye una porción importante del financiamiento a la producción, dado el escaso desarrollo del mercado de capitales (De Nigris, 2008).

En resumen, el estudio del crédito bancario a las PYMEs es de gran relevancia debido al papel clave del crédito en la financiación de la inversión y el desarrollo y por la importancia de las pymes en la creación de valor agregado y empleo. Este trabajo analiza la relación entre el sistema bancario argentino y las PYMEs en el periodo 2002-2009. El trabajo se estructura de la siguiente manera: en primer lugar reseñamos el problema del financiamiento a las PYMEs desde tres enfoques alternativos de la literatura económica: el neoclásico, el basado en las fallas de mercado y el heterodoxo; en segundo lugar, presentamos algunos aspectos relacionados con las particularidades de las PyMEs y su financiamiento; en tercer lugar, analizamos algunos rasgos estilizados del crédito efectivamente otorgado por el sistema bancario en general y la asignación de crédito de los distintos grupos de bancos comerciales -según origen del capital ${ }^{5}$ a las PYMEs en particular

5 Bancos públicos (nacionales y provinciales y municipales) y bancos privados (S.A. de capital nacional, cooperativos, locales de capital extranjero y sucursales de entidades financieras del exterior). 
durante el periodo 2002-2009 en Argentina. ${ }^{6}$ Por último, realizaremos algunas observaciones finales.

\section{EL FINANCIAMIENTO DEL DESARROLLO}

En la actualidad existen tres grandes corrientes teóricas dentro de la literatura económica: la visión neoclásica, el enfoque basado en las fallas de mercado y la heterodoxa. Sus diferencias se basan en los supuestos que cada una de ellas hace sobre cómo funcionan los sistemas económicos, sobre cuál debe ser el rol del Estado en estos sistemas y sobre los factores que condicionan el acceso al financiamiento de las empresas (Kosacoff y Ramos, 1997; Peres y Primi, 2009).

En primer lugar, para la visión neoclásica la estructura de precios brinda a los agentes los incentivos y la información -necesaria y suficiente- sobre la base de los cuales tomar sus decisiones económicas cotidianas; por lo tanto, supone que cualquier intervención en los mercados que afecte los precios relativos distorsionaría la asignación de recursos. La optimización estática de la asignación de recursos que se obtiene a partir de mercados libres conduce entonces a la maximización del crecimiento (Kosacoff y Ramos, 1997). La cuestión del financiamiento del desarrollo se presenta, en general, a partir del análisis de la esfera real de la economía. Se plantea la necesidad de generar inversión para permitir la acumulación de capital y, para ello, el ahorro juega un papel importante. En consecuencia, desde esta teoría se busca determinar cuál es el nivel de ahorro necesario para inducir un proceso de crecimiento. Sin embargo, el estudio de los mercados financieros está menos desarrollado, debido a que la economía neoclásica parte del supuesto de que la actuación de éstos es (y debe ser) neutral. Las instituciones financieras son consideradas simples intermediarias entre ahorristas e inversionistas, con un papel meramente pasivo en la determinación del volumen de fondos para el financiamiento de la inversión (Studart, 2005). El Estado debe velar por el buen funcionamiento del mercado. Siguiendo esta línea de razonamiento, los agentes económicos deberían conseguir recursos para el financiamiento de sus proyectos -cuando éstos sean rentables- $y$,

6 Si bien este trabajo se focaliza en el análisis del crédito efectivamente otorgado a las PYMEs y el comportamiento de los distintos tipos de bancos, entendemos que el estudio de la demanda por parte de las PYMEs es también una parte clave de la problemática. Sin embargo, no realizaremos un análisis exhaustivo de la demanda en este trabajo por limitaciones de tiempo y espacio. 
particularmente, las condiciones de acceso de financiamiento de las PYMEs son similares a cualquier otro proyecto rentable.

En segundo lugar, el enfoque basado en las fallas de mercado, pese a tener una raíz eminentemente neoclásica, deja caer algunos de los supuestos tradicionales. En particular, acepta que los mercados de factores pueden no operar perfectamente y que pueden existir "fallas" en su funcionamiento (Kosacoff y Ramos, 1997). Respecto al mercado financiero en particular, ha surgido una abundante literatura que estudia sus fallas, inexorablemente asociadas a la existencia de problemas de información inherentes a la propia naturaleza de estos mercados. Los trabajos de Stiglitz y Weiss (1981) y Stiglitz et al (1993) son referencias obligadas a la hora de analizar este enfoque. Stiglitz señaló que los agentes económicos no tienen información perfecta sobre todas las opciones y las condiciones de acceso y que los diferentes agentes no son homogéneos entre sí. ${ }^{8} \mathrm{La}$ existencia de estas fallas de mercado puede generar el fenómeno de "racionamiento crediticio". El racionamiento de crédito puede ocurrir en situaciones de información asimétrica en los cuales la tasa de interés no varía para igualar la oferta y la demanda de crédito, sino que se mantiene el precio del crédito a un nivel en el cual la demanda supera a la oferta (Stiglitz y Weiss, 1981). Así, los bancos prefieren mantener el crédito racionado que responder al exceso en la demanda mediante un aumento de precio, porque operan en un entorno caracterizado por "lagunas" en el conocimiento del prestatario y de su proyecto (información asimétrica), porque las tasas altas ahuyentarán a los prestatarios más conservadores (selección adversa) y porque los prestatarios tendrán tendencia a invertir en proyectos más arriesgados si las tasas son más elevadas (riesgo moral) (BID, 2002). Como fue señalado anteriormente, las pyMEs suelen ser los agentes que más padecen este tipo de fenómenos. La conclusión de este grupo de autores es que el Estado debe jugar un rol en los mercados financieros a raíz de la existencia de las fallas de mercado descritas anteriormente (Stiglitz et al, 1993; Kosacoff y Ramos, 1997). ${ }^{9}$ Siguiendo esta línea teórica, se propone la intervención estatal

$7 \quad$ Véase por ejemplo Hallberg (2000).

8 Una de las fallas de mercado más citadas por la literatura es el problema de la información asimétrica que surge de la desigualdad de conocimiento sobre la operación de crédito entre el prestamista y el prestatario. Es decir, el prestatario conoce su negocio y su proyecto mejor que el prestamista, así como el uso que hará de los fondos una vez le sean entregados, y por consecuencia las posibilidades de amortizar el préstamo.

9 También, según este grupo de autores, las imperfecciones provocadas por la información incompleta y asimétrica tienden a ser más pronunciadas cuanto menos diversificados y maduros son los mercados financieros. 
en el mercado financiero para corregir estas fallas de mercado -por ejemplo, a través de una mejora en la cantidad y calidad de la información generada por el sector de pequeñas y medianas empresas en relación al sistema financiero (Arroyo, 2007).

Por último, dentro del enfoque heterodoxo ${ }^{10}$ se enmarcan distintas corrientes que plantean que la racionalidad de la intervención del Estado no se limita a las fallas de mercado, sino que el Estado debe intervenir por las características del sistema económico mismo, y por el reconocimiento de la ausencia de mecanismos de ajuste automáticos. Este enfoque destaca que el aprendizaje tecnológico y el desarrollo de nuevas instituciones constituyen ejes centrales de toda explicación del proceso de desarrollo económico de largo plazo (Peres y Primi, 2009). En consecuencia, los temas centrales van más allá de las tasas de ahorro o de crecimiento óptimas, debiendo focalizarse en cuáles son los cambios estructurales deseables para la economía y qué tipo de infraestructura debe desarrollarse para inducir tales cambios (Kosacoff y Ramos, 1997). En este esquema de pensamiento, el Estado entra en escena como un actor central, guiando la asignación de los recursos en función de una "visión" de óptimo social que trasciende lo que el juego del mercado está en condiciones de ofrecer. Desde esta óptica se señala que tanto las intervenciones funcionales como las selectivas son necesarias para promover el desarrollo, y que los gobiernos son capaces de proveerlas. En ausencia de intervenciones selectivas, la industrialización puede tener lugar, pero su patrón y profundidad se verán afectados, y en los países en vías de desarrollo en la mayoría de las circunstancias tenderá a ser frágil. Así, la teoría predice que la asignación de recursos sería subóptima y el crecimiento se restringiría (Kosacoff y Ramos, 1997).

Si bien dentro de los distintos enfoques reseñados pueden encontrarse contribuciones de relevancia para el estudio del financiamiento de la producción, consideramos que la visión heterodoxa, que es la que incluye miradas propias del desarrollo latinoamericano, es adecuada para estudiar un país con las particularidades de Argentina. Asimismo, se diferencia de los otros enfoques reseñados, que se presentan como modelos de análisis para cualquier tipo de economía -independientemente de sus especificidades.

En este sentido, según la CePAL (1971), la experiencia en América Latina nos muestra que, excepto cuando se han creado expresamente instituciones

10 Dentro de este enfoque agrupamos a las corrientes estructuralistas, poskeynesianas y schumepterianas, ya que tienen una perspectiva común respecto del funcionamiento del sistema económico, más allá de sus diferencias en otros aspectos. 
financieras "desarrollistas" bajo el control del sector público, difícilmente pueda un país resolver los problemas de transferencia intersectorial (o espacial) de recursos hacia los sectores más atrasados, o a nuevos sectores o regiones, mediante el desarrollo espontáneo de sus intermediarios financieros. El movimiento de los recursos desde el sistema financiero a los usuarios de crédito se rige por consideraciones de rentabilidad, liquidez y riesgo de las aplicaciones, y no por sus fines sociales o "desarrollistas". De esta forma, resultan favorecidos los sectores de más alta productividad monetaria y que ofrecen, por lo tanto, colocaciones más "seguras". Según la CEPAL, en América Latina un gran porcentaje de esas operaciones se hallan vinculadas con la financiación de bienes de consumo durables y del capital de trabajo de las empresas, y no con el financiamiento de la inversión real en nuevas actividades o sectores productivos. Para los heterodoxos, el sistema financiero asume un papel activo en la generación y asignación de recursos. Este tipo de funcionalidad del aparato de intermediación promueve una canalización de los recursos diferente de la que tiene lugar en una de carácter "neutro", pues los dirige hacia fines calificados como prioritarios a causa del efecto que tienen en la aceleración del desarrollo económico y social. Estos fines serán distintos, en alta proporción, a aquéllos regidos por el criterio de rentabilidad privada (CEPAL, 1971). En otras palabras, para los heterodoxos es central definir el perfil de crédito requerido en un proceso de desarrollo sostenido en el marco de una economía en vías de desarrollo. En definitiva, las intervenciones en función de superar las "fallas de mercado" no son suficientes para que exista una estructura óptima del desarrollo. El mercado puede funcionar "correctamente", sin embargo se podrían necesitar cambios en el patrón de financiamiento para superar debilidades estructurales. Siguiendo esta línea, es necesario que el Estado disponga efectivamente de los medios e influir cuantitativa y cualitativamente sobre el sistema financiero y sobre los intermediarios individuales, sean públicos o privados (CEPAL, 1971).

Para concluir, cabe destacar que durante los últimos años se han realizado diversas investigaciones que analizan los aspectos centrales de la temática de este trabajo en Argentina. Sin embargo, la mayor parte de la literatura que analiza el vínculo entre el sistema bancario y las PYMEs se inscribe en el enfoque basado en las fallas de mercado. ${ }^{11} \mathrm{Si}$ bien dentro de los distintos enfoques reseñados pueden encontrarse contribuciones de relevancia para el estudio del financiamiento de la producción, este trabajo se inscribe fundamentalmente dentro de la perspectiva heterodoxa.

11 Véase por ejemplo Salloum y Vigier (1999) y Escudé et al. (2001), entre muchos otros. 


\section{LAS PYMES Y EL FINANCIAMIENTO DE LA INVERSIÓN}

Las PYMEs son formas específicas de organización económica en actividades industriales y de servicios en las cuales la producción y el control están en manos de una persona o un grupo que influyen decisivamente en el estilo de gestión, conducción o manejo (Salloum y Vigier, 1999). ${ }^{12}$ Las condiciones particulares de funcionamiento de estas empresas las diferencian de las grandes debido a que cuentan con problemas de escala y menores niveles de productividad, lo cual redunda en menores volúmenes de producción y niveles de rentabilidad. Una hipótesis bastante difundida en relación con las características de las PYMEs de los países de la región es su rezago "cualitativo" en lo tecnológico/productivo, organizativo/gerencial y de mercado en relación con las empresas grandes. Este rezago se traduce en una estructura productiva polarizada o heterogénea, caracterizada por grandes diferenciales de productividad entre las empresas grandes y las de menor tamaño (Held, 1995). Al mismo tiempo, se diferencian de las microempresas -que existen en muchos casos como actividades de subsistencia y como único mecanismo de acceso para la obtención de ingresos familiaresporque su organización responde en términos generales a la lógica de la ganancia capitalista. En este sentido, la problemática de las PYMEs adquiere características propias (CIEPp, 2005).

En este marco, el acceso a diferentes tipos de recursos juega un rol de vital importancia en la determinación del crecimiento de una PYME. Es posible reconocer tres recursos centrales: el financiamiento, la tecnología, y el trabajo y gerenciamiento calificado (Salloum y Vigier, 1999). Como ya señalamos, desde el punto de vista de su financiamiento, las empresas pueden obtener los fondos necesarios para su crecimiento de fuentes internas (utilidades retenidas o provenientes de otros sectores vinculados) y externas (sistema bancario, mercado de capitales y crédito comercial de proveedores y contratistas). La empresa que desea mantener o expandir sus operaciones deberá financiarse a través de cualquiera de estas fuentes. La provisión de financiamiento eficiente y efectivo ha sido reconocida como factor clave para asegurar que aquellas firmas con potencial de crecimiento puedan expandirse y ser más competitivas (Elosegui et al, 2007; Yoguel, 1999).

12 Cabe señalar que no existe una única definición de pyme debido a la existencia de distintos criterios para encuadrar a una empresa dentro esta categoría. Los criterios pueden variar no sólo entre distintos países sino también dentro de un mismo territorio a distintos efectos (IDEPYME-ABAPPRA, 2004). 
La explicación de este fenómeno se basa en múltiples razones. En la literatura se enumeran cuatro componentes principales que condicionan el acceso al financiamiento de largo plazo de las empresas y su utilización: en primer lugar, los factores macroeconómicos que dificultan la conformación de un mercado de crédito de largo plazo; en segundo lugar, los factores institucionales específicos que afectan al sistema financiero; en tercer lugar, las características particulares de las empresas potencialmente receptoras de financiamiento; por último, las características especiales de las entidades financieras potencialmente oferentes de financiamiento (Anastasi et al, 2008). ${ }^{13}$ En consecuencia, el problema del financiamiento adquiere distinta relevancia según el tamaño de la empresa -para las empresas pequeñas o medianas la capacidad de acumular beneficios puede ser más acotada respecto a las grandes. Así, el acceso al financiamiento por fuentes externas pasa a ser prioritario para el proceso de expansión de las empresas PYMEs.

\section{EL FINANCIAMIENTO BANCARIO DE LAS PYMES EN LA POSCONVERTIBILIDAD (2002-2009)}

\section{1. Una mirada global sobre el crédito bancario en Argentina}

A continuación presentamos algunas características del crédito del sistema bancario argentino de las últimas décadas.

El crédito bancario total expresado como porcentaje del PBI (Gráfica 1) desciende sostenidamente luego de la crisis del 2001, pasando de representar el $44 \%$ del pBi en el año 2002 a $21 \%$ en el año $2009 .{ }^{14}$ Durante el periodo 20022009 -y considerando los valores en términos corrientes- los préstamos bancarios crecieron un $82 \%$, mientras que el PBI y la IBIF crecieron $268 \%$ y $541 \%$

13 En el caso específico de la problemática del financiamiento a las PYMEs influyen distintos factores tales como el alto costo del crédito, el reducido tamaño del negocio, la insuficiente disponibilidad de garantías adecuadas, la preferencia por el uso de recursos propios, el limitado volumen y la escasa diversificación de sus operaciones bancarias, la escasa disponibilidad de personal capacitado en los bancos para atender al segmento pyme, los elevados costos de transacción en las operaciones con estas firmas, la falta de confianza de los bancos respecto de sus proyectos, la falta de transparencia contable, la pérdida de peso de la banca especializada en el crédito pyme, y las restricciones de acceso al mercado de capitales que enfrentan estas firmas, entre otros (BID, 2002; Bleger y Rozenwurcel, 2000).

14 Sobre el nivel de bancarización en Argentina véase De Nigris (2008). 
Gráfica 1. Crédito bancario total y crédito bancario al sector público y privado como porcentaje del PBI (1993-2009)

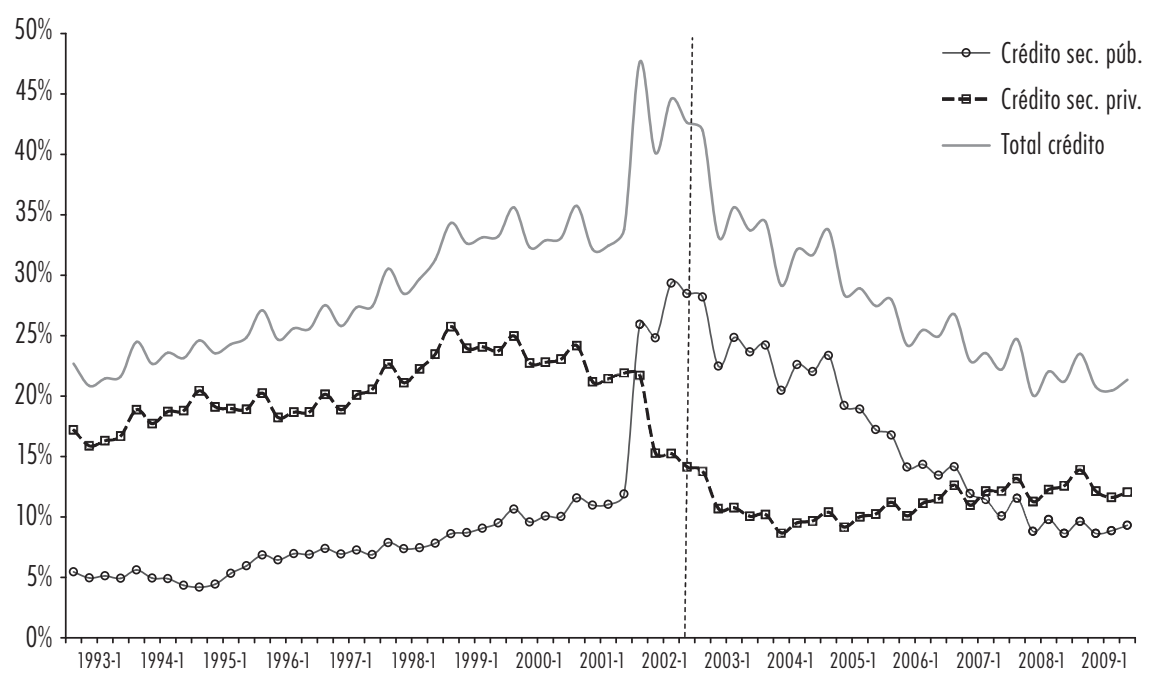

Fuente: elaboración propia con base en BCRA y MECON.

respectivamente. Respecto a la desagregación del crédito del sistema bancario a los sectores privado y público respectivamente, como porcentaje del PBI, se observa que el crédito al sector privado experimenta una caída brusca durante la crisis y luego una recuperación muy tenue, sin alcanzar los niveles de los 90. El ratio crédito bancario al sector privado/PBI promedio considerando el periodo 2002-2009 fue 12\% -considerablemente inferior al promedio de la década anterior (20\%)- muy inferior al de los países desarrollados ${ }^{15}$ e inclusive inferior al de ciertos países comparables por su nivel de desarrollo (De Nigris, 2008). El crédito al sector público aumenta considerablemente durante la crisis, decreciendo luego lentamente hasta alcanzar en la actualidad niveles similares a los picos de la década anterior. En el periodo posconvertibilidad, el crédito al sector público excedió al privado hasta el 2007, año a partir del cual el crédito al sector privado pasa a ser mayor-aunque por una diferencia mínima. A partir de estos datos, podemos

15 Por ejemplo, para Japón el ratio crédito bancario al sector privado/PBI fue 101\%, para EEUU $55 \%$ y para Canadá 122\% considerando el periodo 2002-2007. 
suponer la existencia de una significativa tasa de autofinanciamiento por parte de las empresas locales. Esta tasa se mantiene elevada aun años después de la crisis y con el sistema bancario "normalizado". En el caso de las PYMEs, el elevado nivel de autofinanciamiento puede considerarse como una característica estructural histórica de Argentina, producto de periodos y fases de alta restricción crediticia por el lado de la oferta, y gran inestabilidad macroeconómica cíclica que afectan las condiciones de demanda. Si bien desde el punto de vista de la economía empresaria, el autofinanciamiento se puede plantear como la estructura de plazo y costo óptima en términos de ciertos agentes económicos, a nivel macroeconómico presenta importantes limitaciones (Golonbek, 2008).

Gráfica 2: Financiaciones por destino como porcentaje del total de financiaciones (1990-2009)

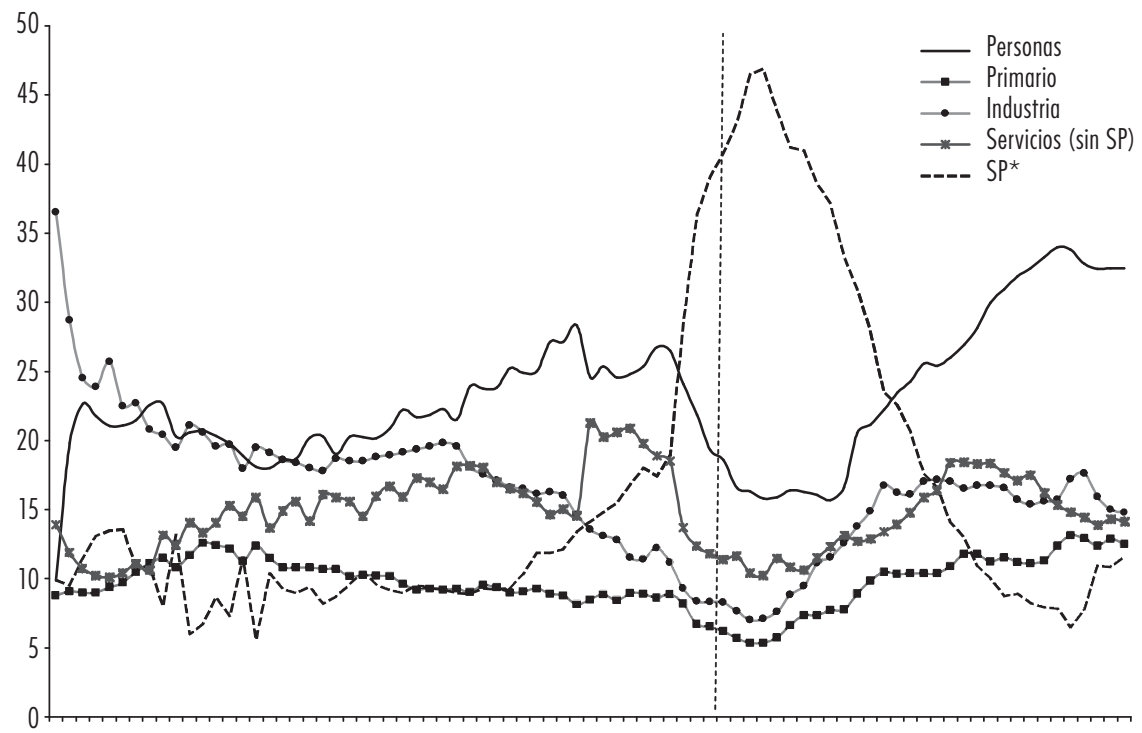

19009-1

* Administración pública, defensa y seguridad social obligatoria.

Fuente: elaboración propia con base en BCRA.

Adicionalmente, consideramos relevante examinar el financiamiento al sector privado no financiero (SPNF) por tipo de actividad (Gráfica 2). ${ }^{16}$ En el

16 Estos datos corresponden a las financiaciones otorgadas por el sistema financiero total y no exclusivamente por el sistema bancario dado que no existen datos desagregados. 
periodo 2002-2004, la participación del sector público en las financiaciones llegó a superar el 45\% del financiamiento total, a la vez que los sectores restantes (personal, primario, industria y servicios) disminuyeron su cuota. A partir del año 2004, las financiaciones al SPNF aumentaron sostenidamente su participación con respecto al sector público; sin embargo, se destaca la marcada participación creciente de los créditos personales, que pasaron de representar el $19 \%$ de las financiaciones en el año 2002 al 33\% en el año 2009, alcanzando hacia el final del periodo en estudio valores que superan ampliamente los más altos de la década de la convertibilidad. Esto refleja una tendencia creciente del crédito de corto plazo priorizando el consumo en detrimento del crédito a los sectores productivos, tanto primarios como manufactureros, en consonancia con el análisis heterodoxo, que plantea que el movimiento de recursos financieros se rige principalmente por condiciones de rentabilidad, liquidez y riesgo de las aplicaciones (véase sección 2).

Cuadro 2. Evolución de la cantidad de entidades bancarias (a diciembre de cada año)

\begin{tabular}{|c|c|c|c|c|c|c|c|c|c|}
\hline \multirow[b]{2}{*}{ Año } & \multirow[b]{2}{*}{$\begin{array}{c}\text { Total } \\
\text { bancos }\end{array}$} & \multicolumn{3}{|c|}{ Bancos Públicos } & \multicolumn{2}{|c|}{ Bancos Privados } & \multirow[b]{2}{*}{ Cooperativos } & \multirow[b]{2}{*}{$\begin{array}{c}\text { Capital } \\
\text { Extranjero }\end{array}$} & \multirow[b]{2}{*}{$\begin{array}{c}\text { Suc. de } \\
\text { Entidades } \\
\text { Extranjieras }\end{array}$} \\
\hline & & Total & Nacionales & $\begin{array}{c}\text { Provinciales } \\
y \\
\text { Municipales }\end{array}$ & Total & $\begin{array}{l}\text { Capital } \\
\text { Nacional }\end{array}$ & & & \\
\hline 1991 & 167 & 35 & 6 & 29 & 132 & 66 & 38 & & 31 \\
\hline 1995 & 135 & 33 & 4 & 29 & 102 & 59 & 12 & & 31 \\
\hline 2000 & 89 & 14 & 2 & 12 & 75 & 34 & 2 & 21 & 18 \\
\hline 2005 & 71 & 13 & 3 & 10 & 58 & 34 & 1 & 12 & 11 \\
\hline 2009 & 66 & 12 & 2 & 10 & 54 & 32 & 1 & 12 & 9 \\
\hline
\end{tabular}

Fuente: elaboración propia con base en BCRA.

Por último, a partir de los datos de evolución de la cantidad de entidades bancarias se revela que el proceso de concentración y extranjerización del sistema bancario argentino durante la década de $1990^{17}$ determinó la desaparición de bancos tradicionalmente ligados a financiar el segmento PYME (como los bancos cooperativos); este proceso no se ha revertido en la posconvertibilidad.

17 Véase Cibils y Allami (2010). 
Este fenómeno -entre muchos otros- determinó que una gran porción de las PYMEs no pudieran sobrevivir.

Adicionalmente, la lógica de la banca internacional permeó la operatoria de la banca local pública y privada, acentuando las tendencias que se verifican a nivel internacional (crédito de corto plazo orientado al consumo, fuerte aumento de la renta por servicios financieros, etcétera). ${ }^{18}$ Este proceso, que tuvo un marcado impacto sobre el sistema bancario en la década de la convertibilidad, ha tendido a acentuarse en el periodo posterior a la crisis, dando mayor relevancia a la crítica heterodoxa de la liberalización financiera.

\section{Los créditos bancarios a las PYMEs}

En el presente apartado presentamos algunos datos sobre la evolución y estructura del financiamiento bancario a las PYMEs en el periodo de la posconvertibilidad. Cabe destacar que no existen estadísticas precisas sobre la situación del financiamiento a las PyMEs en Argentina. En esta propuesta de investigación se utilizará la metodología considerada por García (2006) y el CEFID-AR ${ }^{19}$ que consiste en un cálculo estimado con base en los datos de préstamos del sistema bancario por tramos. ${ }^{20}$

Como se observa en la Gráfica 3.a, los créditos a las PYMEs -en valores corrientes - tienen una tendencia ascendente a partir del año 2003. Esta tendencia también se observa considerando la desagregación entre bancos públicos y privados, aunque a partir del año 2009 la banca pública mantiene una tendencia creciente mientras que la banca privada revierte la tendencia.

En el detalle del crédito por tipo de banco según origen del capital, observamos que, medidos en valores corrientes, los préstamos a las PYMEs de los bancos privados de capital nacional han sido los más relevantes durante el periodo 2004-2009, seguidos de los bancos privados locales de capital extranjero.

18 Véase, por ejemplo, Dos Santos (2009).

19 Centro de Economía y Finanzas para el Desarrollo de la Argentina.

20 El CEFID-AR realiza una estimación trimestral que consiste en sumar a) la cartera comercial correspondiente a los préstamos menores a 500.000 pesos; $b$ ) los préstamos correspondientes a los tramos de entre 500.000 y 5.000 .000 pesos. Tal como lo seńala Kulfas (2008), este criterio tiene aspectos cuestionables, en tanto pueden existir empresas grandes que tengan saldos de deuda inferiores a esa cifra, aunque seguramente serán pocas las PYMEs que puedan tener saldos de deuda superiores a ese monto. 
Gráfico 3.a. Evolución del financiamiento bancario a las PYMEs total y por tipo de banco a julio de cada año (20012009). En millones de pesos corrientes

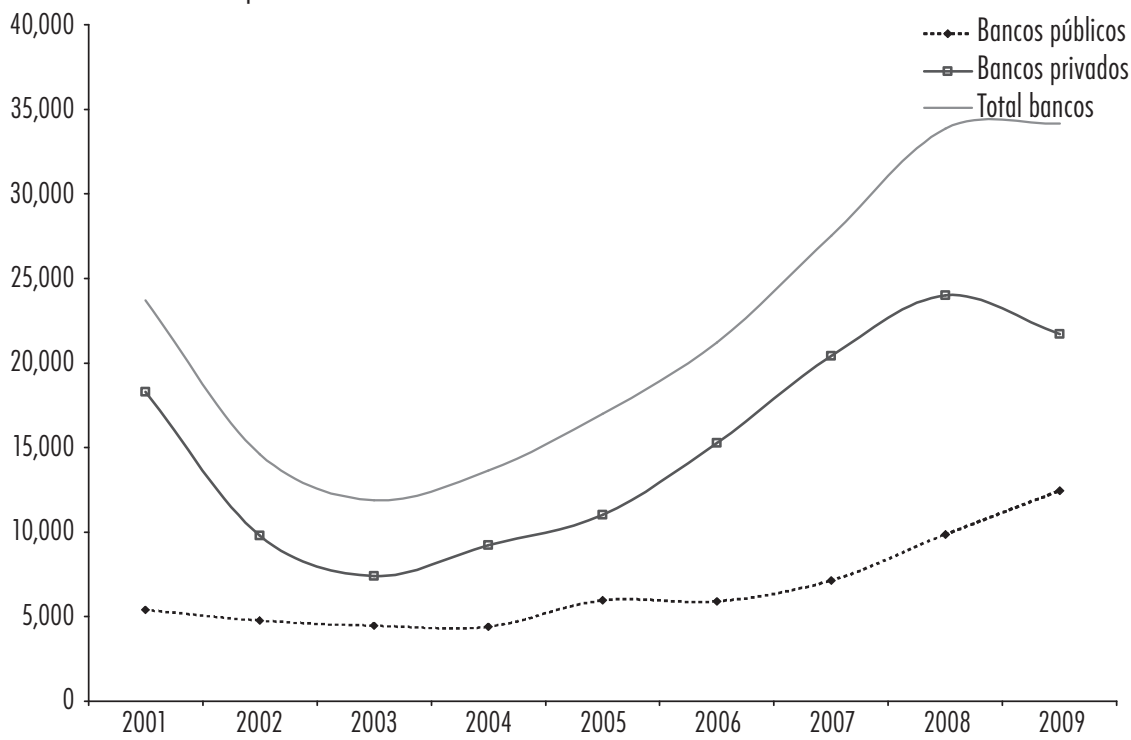

Fuente: elaboración propia con base en BCRA.

Gráfica 3.b. Evolución del financiamiento bancario a las PYMEs por tipo de banco según origen del capital. A julio de cada año (2001-2009). En millones de pesos corrientes

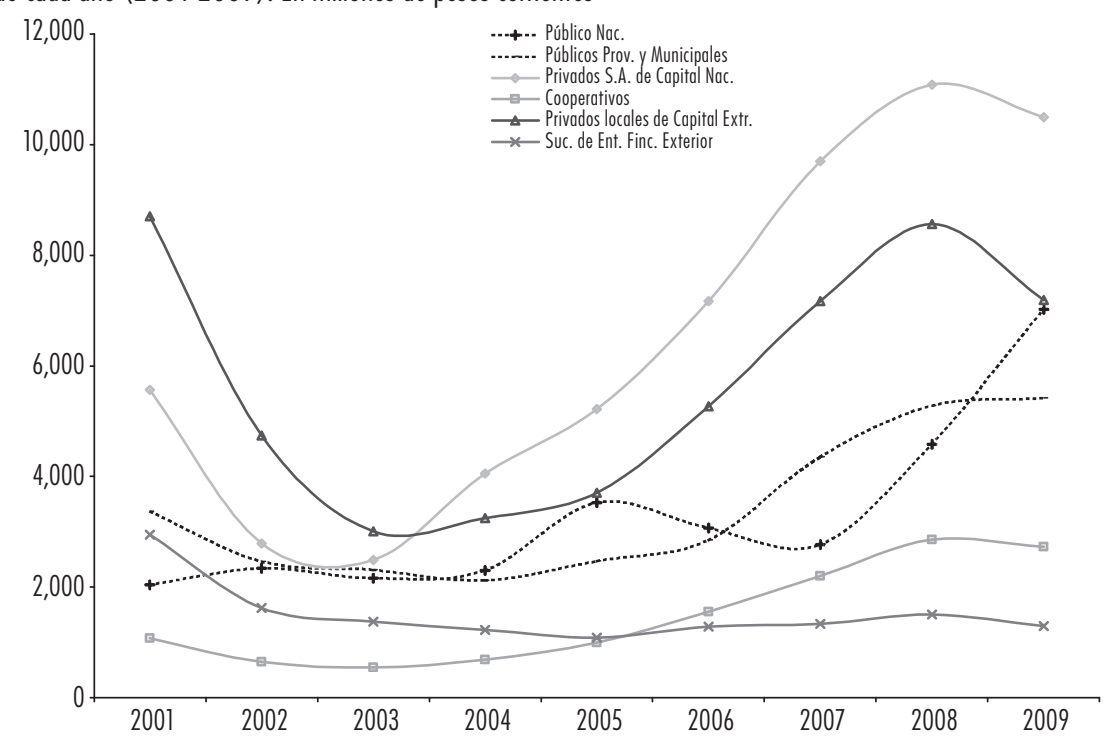

Fuente: elaboración propia con base en BCRA. 
Los bancos públicos nacionales incrementaron sustancialmente el monto del financiamiento a partir del año 2007. Los bancos cooperativos, si bien con montos menores, muestran un claro aumento en el crédito a las pyMes, triplicando el valor en términos corrientes en el periodo bajo estudio. Por su parte, las sucursales de entidades financieras del exterior son los bancos que menores montos -en valores absolutos- han destinado al financiamiento a PYMEs, mostrando incluso una leve tendencia decreciente en todo el periodo. Cabe señalar que, durante el año 2008 -cuando la crisis internacional impacta en la Argentina-, la banca pública nacional aumentó notablemente su financiamiento a las PYMEs, mientras que la banca privada nacional y extranjera redujo su financiamiento al sector.

Gráfica 4.a. Créditos a PYMEs, total y por tipo de banco como porcentaje del total de créditos otorgados. A julio de cada año (2001-2009)

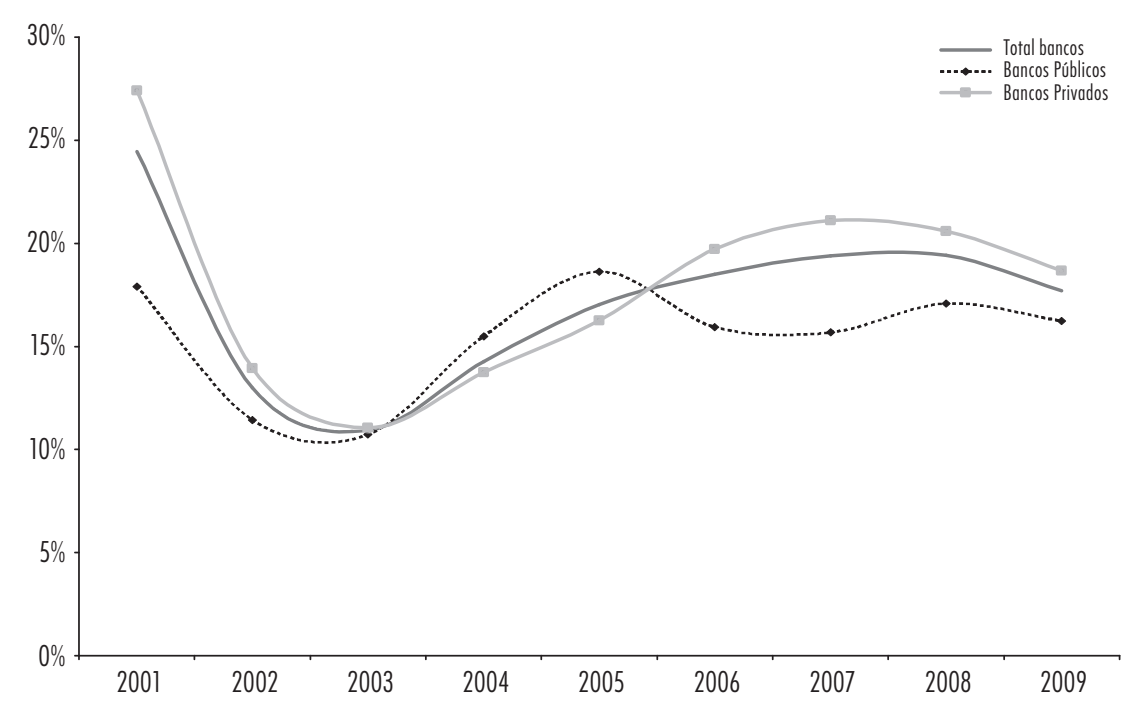

Fuente: elaboración propia en base en BCRA.

En la Gráfica 4.a presentamos la evolución del financiamiento a las PYMEs como porcentaje del total de créditos bancarios (total y de cada grupo de entidades bancarias). Como se observa en la gráfica, durante el periodo 2003-2007 se incrementa el crédito a PYMEs como porcentaje del total. En el caso de los 
bancos privados se observa la misma tendencia, mientras que los bancos públicos muestran una tendencia ascendente en los periodos 2003-2005 y 2007-2008 y descendente en el periodo intermedio. En resumen, los bancos incrementaron sostenidamente la participación de los créditos a las PYMEs como proporción de su cartera total de préstamos entre 2003-2007, aunque esta tendencia parecería haberse revertido en los últimos años, tanto para la banca pública como privada.

Gráfica 4.b. Créditos a PYMEs por tipo de banco según origen del capital como porcentaje del total de créditos otorgados a julio de cada año (2001-2009)

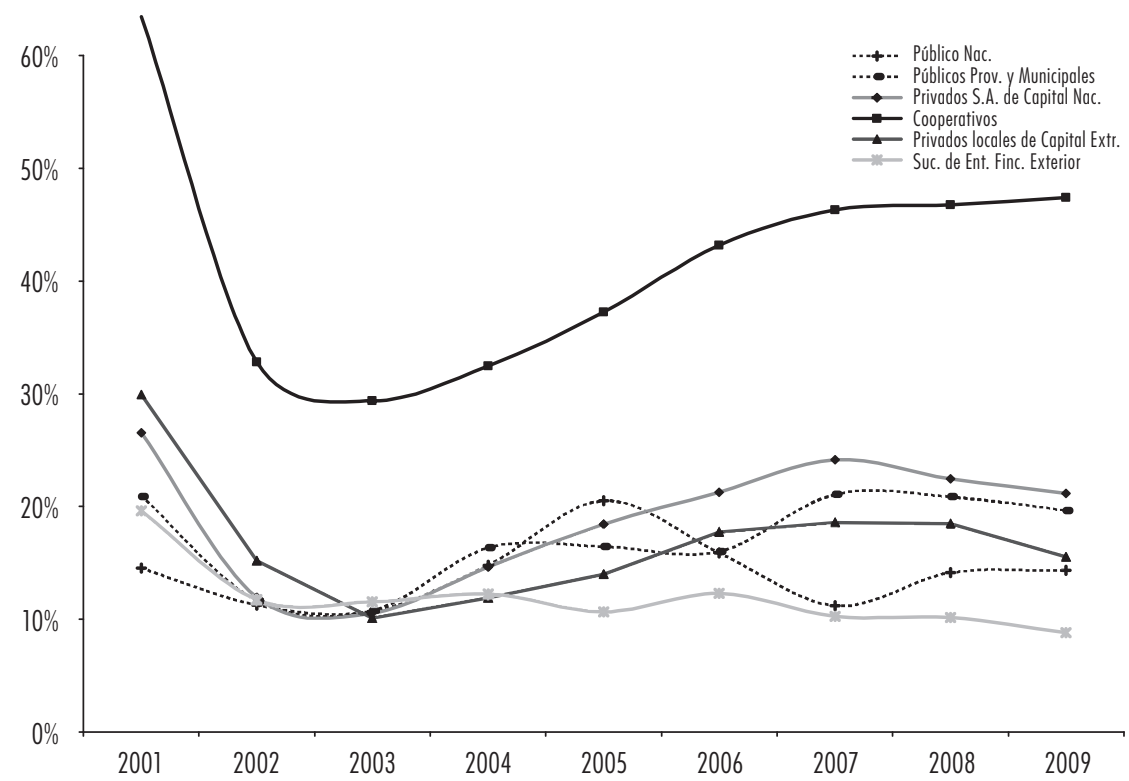

Fuente: elaboración propia con base en BCRA.

Considerando el mismo indicador por grupos de bancos según origen del capital (Gráfica 4.b), observamos que los bancos cooperativos son los que más proporción de su cartera destinan a crédito a pyMes. A partir del año 2003 la participación del crédito a PYMEs en la cartera de la banca cooperativa se ha incrementado sostenidamente, pasando de $29 \%$ en el año 2003 a $47 \%$ en el año 2009. La cartera de créditos a PYMEs promedio de estos bancos considerando el periodo 2002-2009 fue de 39\%. Por otro lado, la cartera pyME de los bancos 
públicos nacionales ha sido variable, con un ratio promedio de $14 \%$ en el periodo 2002-2009. Esta misma tendencia oscilante se observa en el caso de los bancos públicos provinciales y municipales, cuya cartera PYME osciló en promedio en torno al $17 \%$ de los préstamos totales. La cartera PYME correspondiente a los bancos privados de capital nacional se incrementó sostenidamente hasta el año 2007 -año en el que alcanzó un pico de 24\%. Las sucursales de las entidades financieras del exterior son los bancos que destinan menor proporción de sus créditos a PyMEs ( $11 \%$ en promedio 2002-2009), con tendencia decreciente en todo el periodo bajo estudio.

Gráfica 5.a. Créditos a PYMEs, total y por tipo de banco como porcentaje del total de créditos otorgados al SPNF. A julio de cada año (2001-2009)

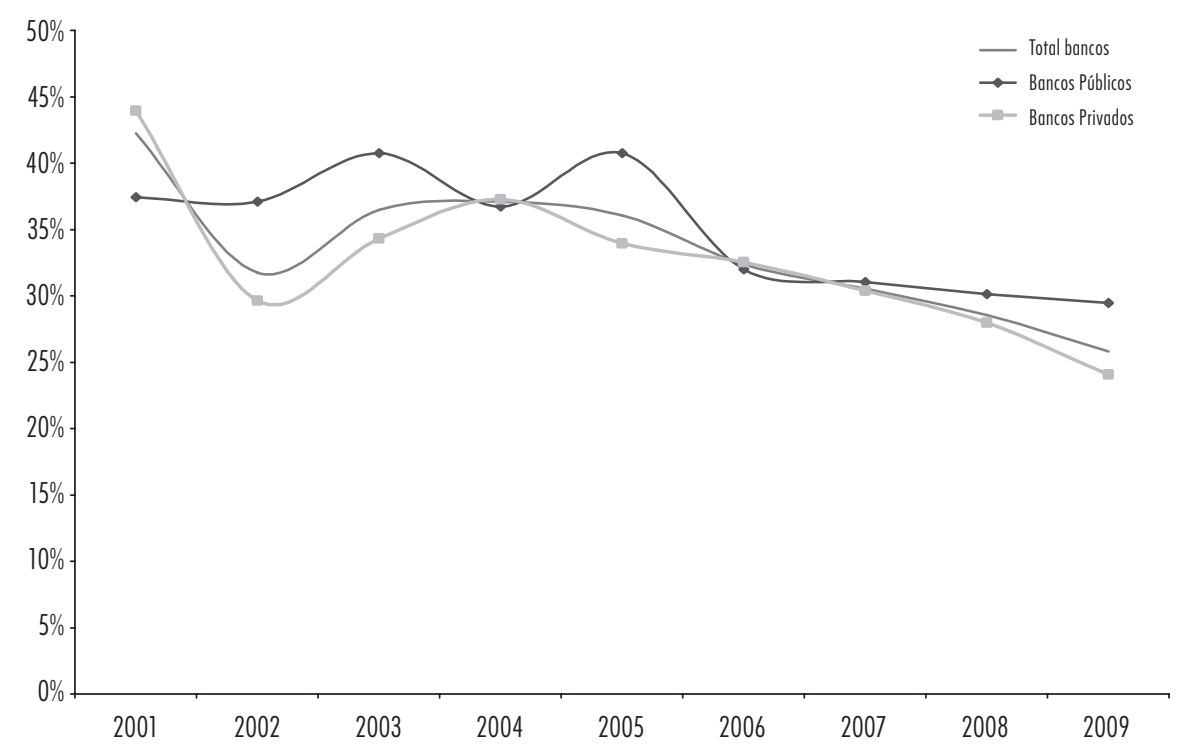

Fuente: elaboración propia con base en BCRA.

Otro indicador que consideramos relevante es la proporción de préstamos a las PYMEs como porcentaje del total de préstamos al SPNF. En este caso, se observa una tendencia descendente a partir del ańo 2004, tanto para los bancos públicos como los privados. En el año 2006, los bancos destinaron en promedio $26 \%$ de los préstamos a PYMEs considerando el total de préstamos al SPNF. 
Gráfica 5.b. Créditos a PYMEs por tipo de banco según origen del capital como porcentaje del total de créditos otorgados al SPNF a julio de cada año (2001-2009)

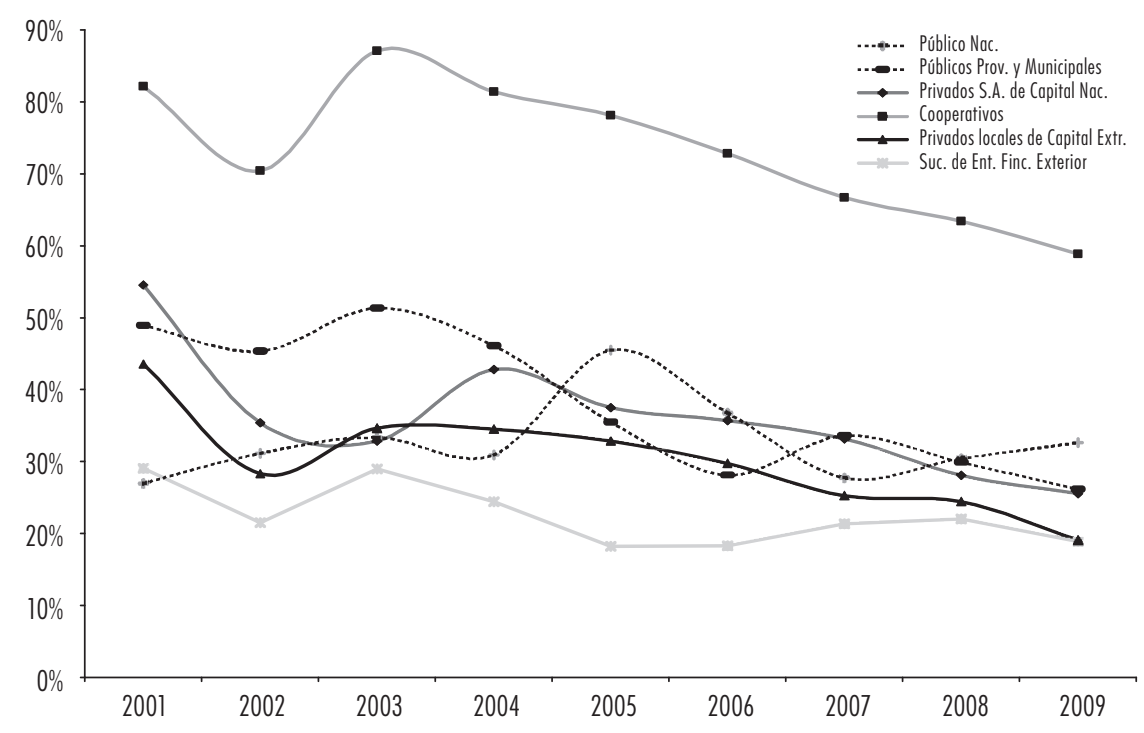

Fuente: elaboración propia con base en BCRA.

En el detalle por tipo de bancos según origen del capital, observamos que la tendencia descendente anteriormente seńalada se replica para todos los grupos de bancos -excepto para los públicos nacionales, para los cuales el ratio se mantiene en promedio relativamente constante en torno al 33\% en 2002-2009. Los bancos cooperativos son los que cuentan con una mayor participación de créditos a PYMEs en relación a los préstamos totales al SPNF (promedio de $72 \%$ del total de sus préstamos al SPNF en el periodo 2002-2009). Los bancos públicos provinciales y municipales disminuyen marcadamente la participación de los créditos a PYMEs en su cartera de préstamos al SPNF, pasando de $51 \%$ en el año 2003 a 26\% en el año 2009. Los bancos privados de capital nacional tuvieron una participación promedio de préstamos a PYMEs de $26 \%$ en su cartera, mientras que para las sucursales de entidades financieras del exterior el crédito promedio a PYMEs fue de $19 \%$.

Con base en el análisis de estos datos podemos concluir que si bien la relación créditos a PYMEs/créditos totales tuvo una tendencia ascendente durante la posconvertibilidad hasta el año 2007, si consideramos la relación créditos a PYMEs/créditos al SPNF la relación es levemente descendente durante todo el 
periodo, por lo que podemos inferir que los créditos personales (el componente del crédito al SPNF con mayor crecimiento) puede estar ganando participación a costa del crédito a las pYMEs. En general, los créditos personales representan operaciones de muy alta rentabilidad para los bancos, al margen del origen de su capital. Además, observamos que el peso de la cartera de créditos a PYMEs varía según el grupo de bancos por origen del capital; si bien los bancos privados de capital nacional son los que han provisto los mayores volúmenes de financiamiento a PYMEs en la posconvertibilidad no tienen una orientación comercial marcada hacia ese segmento de empresas. Por otro lado, los bancos cooperativos han destinado un porcentaje relevante de su cartera de créditos a PYMEs; sin embargo, el monto de créditos otorgado es marginal si consideramos el volumen total de créditos.

\section{Una mirada desde la demanda de financiamiento de las PYMEs}

A continuación, y como complemento al análisis de la oferta de crédito bancario presentado en la sección anterior, analizaremos algunos rasgos estilizados de la demanda de financiamiento de las PYMEs en la posconvertibilidad. ${ }^{21}$

Cuadro 3. Fuentes de financiamiento de las inversiones de las PYMEs industriales

\begin{tabular}{lrrrrrrr}
\hline & 1998 & 2003 & 2005 & 2006 & 2007 & 2008 & 2009 \\
\hline Recursos propios & 51,6 & 87,7 & 83 & 77,2 & 65,8 & 60,8 & 63,6 \\
Financiamiento bancario & 26,7 & 4,4 & 8,2 & 14 & 21,8 & 24,6 & 21,3 \\
Finnanciamiento de proveedores & 18,5 & 6,4 & 7,2 & 5 & 7,8 & 7,8 & 7,9 \\
Financiamiento de clientes & - & 0,1 & 0,8 & 1,4 & 1,6 & 2,3 & 1,4 \\
Programas públicos & 0,1 & - & - & 1 & 2 & 1,4 & 2,8 \\
Otras financiaciones & 3,1 & 1,3 & 0,8 & 1,4 & 1 & 3 & 3 \\
\hline
\end{tabular}

Fuente: Fundación Observatorio PYME (1998-2010).

21 Los datos de esta sección se basan en los informes elaborados por la Fundación Observatorio PYME con base a una encuesta a PYMEs industriales (Fundación Observatorio PYME, 1998-2010). 
La principal fuente de financiamiento de las PYMEs industriales para realizar inversiones son los recursos propios, tal como se observa en el Cuadro 3. La segunda fuente en importancia de financiamiento son los créditos bancarios. Si bien el crédito bancario ha incrementado su participación luego de la fuerte caída en la crisis de 2001-2002, todavía no se alcanzaron los niveles de participación de financiación bancaria del año $1998,{ }^{22}$ y sigue siendo muy inferior al uso de recursos propios.

Cuadro 4. Proyectos de inversión frenados por falta de financiamiento bancario

\begin{tabular}{lcccccc}
\hline & 2004 & 2005 & 2006 & 2007 & 2008 & 2009 \\
\hline $\begin{array}{l}\text { Proporción de empresas con proyecto } \\
\text { frenado por falta de financiamiento } \\
\text { bancario }\end{array}$ & 32.9 & 31.5 & 27.6 & 27.9 & 29.4 & 29.5 \\
\hline
\end{tabular}

Fuente: Fundación Observatorio PYME (2004-2010)

Ahora bien, estos datos no reflejan la existencia de un potencial techo al crecimiento de las pyMes debido a que las empresas podrían estar financiando sus inversiones con recursos propios por ser más conveniente y económico que tomar deuda; sin embargo, si observamos el porcentaje de empresas PyMEs con proyectos de inversión no realizados por falta de financiamiento bancario podemos acercarnos a un indicador de este potencial límite al crecimiento y expansión de estas empresas. En efecto, en el Cuadro 4 observamos que durante el periodo 2004-2009 aproximadamente el 30\% de las PYMEs presentaba proyectos de inversión frenados debido a que no existía disponibilidad de fondos bancarios.

El escaso peso del financiamiento bancario en la estructura de financiación de las PYMEs está vinculado, también, a un fenómeno que opera desde el lado de

22 Si bien no se cuenta con los datos de fuente de financiación de grandes empresas para el periodo analizado, según datos del Banco Mundial -con base en una encuesta realizada a aproximadamente 1000 empresarios de Argentina en el año 2006-, en el ańo 2006 sólo el 3,3\% de las pequeñas empresas utilizaron crédito bancario para financiarse, mientras que para las medianas y grandes el porcentaje correspondiente fue de $8,5 \%$ y $14 \%$ respectivamente. Por otro lado, el $40 \%$ de las pequeñas empresas manifestaron que el acceso al crédito es un problema serio para su crecimiento, mientras que el 38,5\% de las medianas y el 34\% de las grandes afirmaron presentar este obstáculo (Bebczuk, 2010 con base en datos del Banco Mundial). 
la demanda: la autoexclusión de las PYMEs del crédito bancario. En efecto, sólo un $40 \%$ de las pyMEs solicitó algún tipo de financiamiento alguna vez durante el periodo 2003-2006 (Kulfas, 2009) y el 70\% de los empresarios no solicitó créditos de mediano y largo plazo en 2009 (Fundación Observatorio PYME, 2010). Tal como se observa en el Cuadro 5, las razones de esta autoexclusión del mercado de crédito son diversas; sin embargo, en el año 2009 aproximadamente el $40 \%$ de las empresas alegaron motivos vinculados con restricciones históricas de las PYMEs para acceder al financiamiento (altos costos financieros, dificultades para obtener garantías, etcétera).

Cuadro 5. Motivo por el cual las PYMEs industriales no han solicitado crédito bancario distinto al de cuenta corriente $^{23}$ (\% de empresas)

\begin{tabular}{lrrr}
\hline & 2005 & 2006 & 2009 \\
\hline Incertidumbre sobre la evolución de la economía nacional & 23.3 & 32 & 42 \\
Costo financiero elevado o plazo cortos para la cancelación & 25.9 & 35 & 29 \\
El banco no lo califica como sujeto de crédito debido a las deudas fiscales & 4.1 & 9 & 6 \\
No cubre exigencias de garantías & 5.3 & 12 & 5 \\
El banco no lo califica como sujeto de crédito por elevado endeudamiento & 2.7 & 6 & 2 \\
Otra razón & 3.5 & 7 & 15 \\
\hline
\end{tabular}

Fuente: Fundación Observatorio PYME (2006-2010).

\section{OBSERVACIONES FINALES}

Con base en los datos presentados sobre los créditos otorgados a PYMEs por los distintos tipos de entidades bancarias argentinas podemos realizar algunas observaciones generales respecto al financiamiento a las PYMEs durante este periodo.

En primer lugar, la proporción del crédito bancario como porcentaje del PBI -que descendió en la posconvertibilidad respecto a la década del 90- es bajo en relación con los países desarrollados e inclusive inferior al de ciertos países comparables por su nivel de desarrollo. En segundo lugar, la desagregación del crédito al SPNF por destino, como porcentaje del total de las financiaciones, muestra un fuerte y sostenido aumento del crédito personal (de corto plazo, principalmente para el consumo) en detrimento del crédito al sector productivo. En tercer lugar,

23 Los créditos bancarios distintos a los de cuenta corriente corresponden a créditos de mediano y largo plazo. 
el crecimiento de los créditos destinados a las PYMEs no fue proporcional al aporte de estas empresas al crecimiento del producto bruto interno ni de la inversión interna bruta fija, de manera que podemos inferir que estos incrementos se dieron en gran medida con base en el autofinanciamiento. En cuarto lugar, la relación créditos a PYMEs/créditos totales tuvo una tendencia ascendente durante la posconvertibilidad hasta el año 2007, a partir del cual vuelve a declinar. No obstante, si consideramos la relación créditos a PYMEs/créditos al SPNF la relación es levemente descendente durante todo el periodo, por lo que podemos inferir que los créditos personales (el componente del crédito al SPNF con mayor crecimiento) puede estar ganando participación a costa del crédito a las PYMEs. En quinto lugar, observamos que el peso de la cartera de créditos a PYMEs varía notablemente según el grupo de bancos por origen del capital.

Por otro lado, a partir del análisis de algunos rasgos estilizados sobre la demanda de financiamiento de las PYMEs podemos deducir que existe demanda de crédito insatisfecha por parte de estas empresas y que, adicionalmente, un gran porcentaje de PYMEs no demanda crédito al sistema bancario por motivos vinculados a las restricciones históricas de estas empresas para acceder al financiamiento (altos costos financieros, dificultades para obtener garantías, no calificación como sujeto de crédito, etcétera).

En resumen, podemos afirmar que en la Argentina existe un mercado de financiamiento a las PYMEs pero que opera en forma limitada y restringida: en la posconvertibilidad no se ha producido una merma en las dificultades históricas de acceso al crédito por parte de las PYMEs. Consideramos que las PYMEs podrían apropiarse de un mayor porcentaje de los créditos; sin embargo, sin políticas de Estado para el mercado bancario, este porcentaje será destinado a créditos a grandes empresas y/o a créditos personales, debido entre otras razones, a que representan operaciones de mayor rentabilidad y menor grado de incobrabilidad para los bancos comerciales. Además, el proceso de concentración y extranjerización del sistema bancario durante la década del 90 generó fuertes cambios en el patrón de financiamiento a la producción por parte de bancos públicos y privados debido a la desaparición de bancos tradicionalmente ligados a financiar el segmento PYME (como los bancos cooperativos); este proceso no parece haberse revertido en la posconvertibilidad. Por este motivo, es relevante analizar la evolución del crédito para determinar cuánto crédito adicional y de qué tipo (plazo, estructura y tasa de interés) es necesario para que estas empresas puedan seguir creciendo a las tasas actuales. En este sentido, es necesaria la intervención y regulación del Estado en el mercado bancario, así como la redefinición de las políticas de crédito de entidades públicas y otras políticas focalizadas para mejorar el financiamiento de las PYMEs en Argentina. 


\section{BIBLIOGRAFÍA}

Anastasi, Alejandra, Gustavo González Padilla y Gastón Repetto, Argentina 2002-2005: utilización de financiamiento bancario, crédito de largo plazo y sus impactos en la performance microeconómica de las empresas, Buenos Aires, BCRA, Documento de trabajo 2008/24, 2008.

Arroyo, Martín, Information Asymmetries, Credit Rationing and Banking Concentration: the Argentinean Case, MPRA, Paper No 10208, 2007.

Ascúa, Rubén, El proceso de creación de empresas post-crisis 2002 y la capacidad de las empresas jóvenes de acceder a financiamiento en Argentina. Una mirada desde la oferta. Trabajo presentado en el II Congreso de AEDA, Buenos Aires, 2010.

BID, Acceso de las pequeñas y medianas empresas al financiamiento, Grupo DFC, Informe de trabajo, 2002.

Bleger, Leonardo y Guillermo Rozenwurcel, "El sistema bancario argentino en los noventa: de la profundización financiera a la crisis sistémica", en revista Desarrollo Económico, núm. 146, vol. 37, Buenos Aires, 1997.

, "Financiamiento a las PYMEs y cambio estructural en la Argentina: un estudio de caso sobre fallas de mercado y problemas de información", en revista Desarrollo Económico, núm. 157, vol. 49, Buenos Aires, 2000.

CEPAL, "El desarrollo reciente del sistema financiero de América Latina", en José Serra (selección), Desarrollo latinoamericano. Ensayos críticos, México, Fondo de Cultura Económica, 1971.

Cibils, Alan y Cecilia Allami, "El sistema financiero argentino desde la reforma de 1977 hasta la actualidad", en Revista Realidad Económica núm. 249, enero-febrero de 2010.

CIEPP, Asistencia financiera a empresas pequeñas y medianas en Argentina. Los programas dependientes de la SEPYME, mimeo, 2005.

Damill, Mario, Nicolás Salvatore y Lucio Simpson, Diagnóstico y perspectivas del sistema financiero argentino. El sistema financiero de la Argentina bajo el régimen de convertibilidad y la transición a un nuevo modo de intermediación, Buenos Aires, Centro de Estudios de la Situación y Perspectivas de la Argentina (CESPA), 2004.

De Nigris, Alberto, La bancarización en Argentina, Serie Financiamiento del Desarrollo, núm. 204, Unidad de Estudios del Desarrollo, División de Desarrollo Económico-CEPAL, Santiago de Chile, 2008.

Dos Santos, Paulo, On the Content of Banking in Contemporary Capitalism, Londres, School of Oriental and African Studies, Department of Economics, Research on Money and Finance Working Paper 3, 2009. 
Elosegui, Pedro, Emilio Blanco, Demian Paula y Panigo, El impacto asimétrico de las restricciones al financiamiento en Argentina. Comparación por sector, tamaño y origen del capital (1995-2003), Buenos Aires, BCRA, Documento de Trabajo 2007/24, 2007.

Escudé, Guillermo, Tamara Burdisso, Marcelo Catena, Laura D’Amato, George McCandless, y Tomás Murphy, Las MIPyMES y el mercado de crédito en la Argentina, Buenos Aires, BCRA, Documento de trabajo núm. 15, 2001.

Fernández Bugna, Cecilia y Fernando Porta, "Crecimiento reciente. Nuevo régimen sin cambio estructural”, en Revista Realidad Económica, núm. 233, Buenos Aires, enero-febrero de 2008.

Filippo, Agustín, Daniel Kostzer y Diego Schleser, Créditos a PYME en Argentina: racionamiento crediticio en un contexto de oferta ilimitada de dinero, Serie Desarrollo Productivo, CEPAL, Santiago de Chile, 2004.

Frenkel, Roberto, "Una política macroeconómica enfocada en el empleo y el crecimiento", en Revista de Trabajo, Año 1, núm. 1, Buenos Aires, Nueva Época, 2005.

Fundación Observatorio PYME, Informe especial: Situación actual y perspectivas para 2010 de la inversión y el financiamiento de las PyME industriales, Buenos Aires, 2010.

Fundación Observatorio PYME, Evolución reciente, situación actual y desafíos futuros de las PyME industriales, 1998-2008.

García, Alfredo, "La financiación hacia las pymes", en Revista Realidad Económica, núm. 224, Buenos Aires, 2006.

Golonbek, Claudio, Banca de desarrollo en Argentina: breve historia y agenda para el debate, Buenos Aires CEFIDAR, Documento de Trabajo núm. 21, 2008.

Hallberg, Kristin, A Market-Oriented Strategy for Small and Medium-Scale Enterprises, IFC Discussion Paper num. 40, Banco Mundial, Washington, D.C., 2000.

Held, Günter, Políticas de financiamiento de las empresas de menor tamaño: experiencias recientes en América Latina, Serie Financiamiento del Desarrollo, Cepal, Santiago de Chile, 1995.

Katz, Jorge y Bernardo Kosacoff, "Aprendizaje tecnológico, desarrollo institucional y la microeconomía de la sustitución de importaciones", en Revista Desarrollo Económico, vol. 37, núm. 148, Buenos Aires, enero-marzo 1998.

Kosacoff, Bernardo y Adrián Ramos, Consideraciones económicas sobre la politica industrial, Buenos Aires, CePal, Documento de Trabajo núm. 76, 1997.

Kulfas, Matías, Las PYMEs argentinas en el escenario post convertibilidad. Políticas públicas, situación y perspectivas, Santiago de Chile, CEPAL, Documento de proyecto, 2008. 
Peres, Wilson y Annalisa Primi, Theory and Practice of Industrial Policy. Evidence from the Latin American Experience, CEPAL, Serie Desarrollo Productivo núm. 187, Santiago de Chile, 2009.

Salloum, Carlos y Hernán Vigier, "La relación bancos-pyMEs", en Revista de la escuela de economía y negocios, San Martín, Pcia. de Buenos Aires, Universidad Nacional de General San Martín, 1999.

Stiglitz, Joseph E. y Andrew Weiss, "Credit Rationing in Markets with Imperfect Information", en American Economic Review num. 71, junio de 1981. , Jaime Jaramillo-Vallejo y Yung Chal Park, The Role of the State in Financial Markets, World Bank Research Observer, Annual Conference on Development Economics Supplement (1993):19-61, 1993.

Studart, Rogério, "El Estado, los mercados y el financiamiento del desarrollo", en Revista de la CEPAL núm. 85, Santiago de Chile, 2005.

Wray, L. R., What do Banks do? What Should Banks Do? Working Paper num. 612, Levy Economics Institute of Bard College, Annandale-On-Hudson, NY, 2010.

Yoguel, Gabriel, "Las restricciones exógenas: la visión de las firmas", en Yoguel y Moori-Koenig (comps), Los problemas del entorno de negocios. Desarrollo competitivo de las PYMEs argentinas, Los Polvorines, Pcia. de Buenos Aires, Fundes Argentina y Universidad Nacional de General Sarmiento, 1999. 